\title{
El distrito de riego 01 y el desarrollo de la agricultura en Aguascalientes, 1925-1935*
}

EDGAR HURTADO HERNÁNDEZ

Facultad ddde Humanidades/UAZ

Cl presente texto es una aproximación a las condiciones del trabajo agrícola en Aguascalientes en las primeras décadas del presente siglo. No se trata de hacer historia de la propiedad ejidal sino de la pequeña propiedad privada asumida como modelo productivo por el presidente Plutarco Elías Calles. A partir de 1926 en el valle de Aguascalientes se crearon las condiciones políticas para incrementar el aprovechamiento productivo y con ello se sentaron las bases para el sometimiento de la élite propietaria local por el gobierno federal. El interés local se expresa desde 1897 a través de Agustín R. González, la respuesta federal se concretó en la visita del Presidente Elías Calles a la zona en 1925 y el marco legal se propuso con la ley de irrigación de aguas federales de 1926.

\section{ANTECEDENTES}

Por las características orográficas e hidráulicas del territorio de Aguascalientes, sabemos que las condiciones para el desarrollo de la agricultura se podrían desarrollar mejor en el centro y surocciden-

* El presente ensayo es una parte de la tesis que el autor presentó para obtener el grado de Maestro en Estudios Rurales por El Colegio de Michoacán, en noviembre de 1996. 
te del estado, aunque para ello habría que hacer inversiones para mejorar las posibilidades de aprovechamiento de la tierra y campañas para transformar la conducta productiva de sus dueños. En este sentido es ilustrativo que ya desde 1881 Agustín R. González se quejara del abandono de la agricultura en el estado; en su opinión habría que convertirla en el motor generador de desarrollo industrial y comercial:

...lejos de obrar así, se cultiva hoy lo que se cultivaba a principios de siglo... nuestros agricultores, con pocas excepciones, permanecen apáticos espectadores de un mal que palpan... en nuestras haciendas se ven grandes siembras de maíz en terrenos que debían ocupar las cepas [para el cultivo de viñedos]... Aguascalientes está llamado por la naturaleza a ser el Málaga, el Jerez de México.

No en todo el territorio estatal podría desarrollarse la idea anterior. En Calvillo y en el valle central las características orográficas e hidráulicas serían más apropiadas para intentar cultivos cuyo mercado rebasara los límites locales. Al segundo habría que agregarle a su favor que tres años más tarde de lo comentado por Agustín R. González se introdujo el ferrocarril; así en el año de 1884 las vías cruzaron el valle de sur a norte elevando el valor de sus terrenos:

Todos los lugares a los que eran conducidos los granos de la región por los arrieros. como Zacatecas. San Luis, Catorce, a partir de 1884 quedaron comunicados por el ferrocarril... todas las haciendas más importantes tenían a escasos kilómetros una estación de ferrocarril. ${ }^{2}$

En opinión de los hacendados locales, al valle sólo le faltó un elemento para garantizar sus inversiones, una obra capaz de almacenar la cantidad de agua suficiente como para garantizar en tiempo

1. Agustín R. González, Historia del estado de Aguascalientes, México, Instituto Cultural de Aguascalientes, Col. La Tradición, 1992, p. 366.

2. Beatriz Rojas Nieto, La destrucción de la hacienda en Aguascalientes, 1910-1931, México, El Colegio de Michoacán, 1981, p. 45. 
y forma los riegos requeridos por la introducción de cultivos destinados a mercados más amplios. En este sentido es ilustrativo el comentario del Dr. Pedro de Alba:

...[por] los lomeríos, las hondonadas y los valles de Pabellón, Garabato o San Luis de Letras... había pasado repetidas veces... por esos parajes verdes y alegres solamente en la temporada de lluvias, resecos, polvorientos y desolados el resto del año. Al caminar un poco a la vera de río Santiago, llamaba la atención el hecho de que en un trayecto de varios kilómetros corriera su caudal en el fondo de una barranca profunda; tanto los rancheros como los hacendados o los sencillos transeúntes exclamaban: ¡si se pudiera levantar aquí una cortina de calicanto, si se almacenara esta agua que se va, si se pudiera regar aquellos bajíos! ${ }^{13}$

La cita anterior nos permite entender que el gobierno estatal encargara al Ing. Tomás Medina Ugarte la realización del proyecto para construir una gran presa. El estudio técnico se conoció en 1897, el cual comprendía la construcción de una presa que detuviese las aguas del río Santiago, además de la edificación de una cortina de mampostería de 46 metros de altura en el punto conocido como "boquilla del Paixtle", sin riesgo de inundar el pueblo de San José de Gracia. De acuerdo a este proyecto la presa almacenaría hasta $45^{\prime} 000,000 \mathrm{~m}^{3}$ de agua con los que se podrían irrigar aproximadamente 9,630 hectáreas, o sea 1,125 de "nuestras antiguas fanegas de campo".4

Se hicieron esfuerzos para construir esta gran presa con la que se garantizaran las inversiones agrícolas: a un valle fértil, bien comunicado y resuelto el problema del agua para riego sólo le faltaría el trabajo fecundo de los hacendados. En 1899 se constituyó una compañía llamada "empresa irrigadora del valle de Aguascalientes", encabezada por Alejandro Vázquez del Mercado, Ignacio T.

3. Pedro de Alba, "La presa Calles y la agricultura salvadora en el estado de Aguascalientes" en: Irrigación en México. Tomo II, febrero de 1931, número 4. p. 335. 4. Archivo Histórico del Estado de Aguascalientes, Fondo de la Secretaría General de Gobierno, Caja 30, expediente 213. (en adelante AHEA, FSGG.) 
Chávez y Ramiro J. Elorduy, quienes se encargarían de solicitar al gobierno federal la concesión de las aguas, de llevar a cabo la construcción de la presa y de solicitar la participación de accionistas. Se comprometieron acciones por la cantidad de 30,700 pesos con los que el proyecto sería realizable. ${ }^{5}$

Si es cierto que el costo de la cortina, compuertas y canales de irrigación alcanzan una suma respetable, no lo es menos que al cabo de diez años de explotación habrá devuelto con creces el capital invertido... en paralelo al entusiasmo despertado entre los verdaderos amantes del progreso, corrieron los capitales suscritos para llevar a buen término una obra que dará a los accionistas pingües utilidades y asegurará el bienestar y la riqueza del estado ${ }^{6}$

El gobierno estatal estaba persuadido de que la obra no sería costosa y el 28 de diciembre de 1914 solicitó en concesión a la Secretaría de Fomento la cantidad de 100,000,000 de $\mathrm{m}^{3}$ de agua del río Santiago para regar una extensión aproximada de 25 a 30,000 hectáreas de terreno. La respuesta llegó el 8 de enero de 1915 y en ella se solicitó mayor información acerca de la cantidad de agua a extraerse del río.

La información encontrada de este primer intento termina aquí, tal vez la marea revolucionaria detuvo la tramitación del proyecto o tal vez los hacendados se preocuparon más por el peligro que sus propiedades corrían por la ley del 6 de enero de 1915 y por el riesgo de que ésta fuese aplicada en el estado, lo cierto es que sería hasta 1926 cuando la iniciativa recobraría vigor pero ahora con la injerencia del gobierno de Plutarco Elías Calles y en un contexto estatal de difíciles relaciones de la élite local con el presidente de la república.

No obstante, a decir del Dr. Pedro de Alba el proyecto no se olvidó. Hasta 1926 se alimentó la ilusión entre sus posibles beneficiarios

5. Archivo Histórico del Agua, Fondo Consultivo Técnico, Caja 4358, exp. 57821, fojas 3 a 23. (en adelante AHA, FCT).

6. AHEA,SGG, саја 30, exp. 213. 
en artículos periodísticos y en conversaciones entre los hombres del poder local; se mantuvo vivo hasta que el General Calles se interesó por él, gracias la intervención decisiva del Dr. Enrique Osornio, quien

...logró que el general Calles hiciera una visita a la región. Con mirada penetrante y con clara inteligencia, se dio cuenta el entonces presidente de que allí podía desarrollarse uno de los aspectos de su vasto plan de obras de irrigación y desde luego ofreció su apoyo para que la obra se realizara... al día siguiente de la gira del Presidente Calles, el Sr. Azpeitia, gobernador interino de Aguascalientes, enviaba a su hijo con un legajo que contenía todos los estudios y proyectos anteriores para que yo [el Dr. Pedro de Alba] los entregara a la presidencia de la república... desde ese momento se podía considerar la gran obra en marcha. ${ }^{2}$

Fue así que en 1926 se iniciaron los trabajos para construir la presa Calles, pero ahora el ingeniero Sellew calculó que el río Santiago podría suministrar anualmente a la presa sólo 294 millones de $\mathrm{m}^{3}$, con los que se podrían irrigar hasta 19,000 hectáreas. ${ }^{8} \mathrm{El}$ sitio señalado para construir la cortina de hasta 63 metros de altura, que necesitó 46,000 metros cúbicos de cemento, se localizó en un cañón angosto y profundo que comunica al terreno de la mesa alta de San José de Gracia con el valle central del estado, un poco abajo del pueblo de San José de Gracia a la entrada del cañón. La presa no se construiría a su salida, como lo sugirió el ingeniero Tomás Medina Ugarte 29 años atrás, por lo que la diferente localización implicó inundar con sus aguas el asentamiento del pueblo:

...dentro del vaso de almacenamiento el trabajo fue particularmente laborioso por haber necesidad de tomar en detalle las construcciones existentes en cada finca rústica y especialmente por estar comprendidos los numerosos pequeños predios, tanto rústicos como urbanos del pueblo de San José de Gracia... se deslindaron dentro del vaso siete propiedades de considerable extensión,

7. Pedro de Alba, op. cit., p. 335

8. Comisión Nacional de Irrigación. Sistema Nacional de Riego "Presidente Calles" (Rio Santiago, Aguascalientes). Memoria Descriptiva formada por el Ing. Manuel Balancari, Supervisor Técnico de las obras. México, Editorial Cultura, 1930, pp. 14-16. 
459 lotes rústicos de San José y 505 predios urbanos en este pueblo que formaba 53 manzanas.?

\section{El Valle DE Aguascalientes}

El terreno entre las sierras de Tepezalá y la de Pabellón y Guajolotes es lo que conocemos como valle de Aguascalientes. Presenta una forma alargada con pendiente suave de norte a sur y de oriente a poniente, con altitudes de hasta 2,000 metros en su extremo norte y de 1,750 metros en su extremo sur. Los terrenos más bajos son los cercanos al cauce del río Aguascalientes o San Pedro, ${ }^{10}$ los terrenos planos van de cada lado hacia el río, en el norte y noroeste del valle son algo ondulados formando colinas que se cortan al toparse con los arroyos del Saucillo y de Rincón, que son sus afluentes. Abajo de ellos y siguiendo su cauce natural, entre los ríos $\mathrm{Pa}-$ bellón y Santiago, los terrenos para cultivo tienen una pendiente de noroeste a sureste, y por último al sur y sureste las pendientes son suaves terminando también el cauce del río. El valle de Aguascalientes mide aproximadamente $70 \mathrm{~km}$. de largo y entre 10 y $20 \mathrm{~km}$. de ancho.

El río fue la principal corriente de agua permanente en el valle, es poco profundo pero de regular anchura. Por su margen occidental recibió las aguas del río Pabellón, del río Santiago y del río Morcinique; por su margen oriente las del río Chicalote, que a su vez estuvo formado por los arroyos de La Dichosa, El Cedazo, Montoro y Peñuelas. Se utilizaron las aguas del río para riego desde la época colonial almacenándose en pequeñas presas, norias y tanques que fueron obra de capitales privados y que no irrigaron la mayor parte de los terrenos del valle. ${ }^{11}$

9. Idem, p. 23

10. Se llama San Pedro porque nace en la sierra de Trancoso del municipio zacatecano de San Pedro Piedra Gorda. Dentro del estado recibe el nombre de río Aguascalientes. 11. АНА, FCT, саja 7, exp, 51, foja 62 . 
Además del mencionado río existieron en el valle aguas freáticas con una profundidad variable de uno a tres metros: hacia su centro aguas confinadas en estratos generalmente arenosos; aguas ascendentes profundas hasta de 240 metros; corrientes subterráneas en cauces sepultados y aguas circulantes que dieron origen a manantiales temporales. El río Aguascalientes y la aguas mencionadas serían susceptibles de utilizarse para riego mediante diferentes obras como: presas, perforación de norias, pozos profundos, galerías filtrantes y socavones. ${ }^{12}$

En los estudios hechos por la Comisión Nacional de Irrigación antes de la construcción del sistema de riego, se calcularon 50,000 hectáreas cultivables de los terrenos del valle y se clasificaron en cinco tipos:

1. Los que ocupaban las vegas de los ríos, profundos en más de 2 metros, ricos y altamente productivos. Su mayor concentración estaba en la unión del río Chicalote con el río Aguascalientes, en los terrenos de Jesús María y entre los ríos de Santiago y Pabellón. Las fajas angostas que ocupan al lado de las corrientes no fueron de gran extensión, pero fueron los mejores terrenos del valle. Midieron aproximadamente 4,000 has.

2. Terrenos buenos pero algo accidentados, que con dificultad son penetrados por el agua. Se encontraron en fajas angostas en las planicies cercanas al río Santiago y al oeste del río Aguascalientes, fueron 2,000 has. en el valle y su productividad fue de entre el 50 y $60 \%$ en relación a las del primer grupo. 3 . Tierras que impidieron la penetración de raíces y agua con espesor de entre 45 y $75 \mathrm{cms}$. Ocuparon 22,500 has. del valle y se encuentran al noreste del río principal y en los llanos noreste y sureste de San Francisco. Fueron suelos deslavados y con fuertes pendientes que pudieron ser utilizados fundamentalmente para pastos. Su productividad fue del $40 \%$ en relación al primer grupo.

4. Suelos con espesor entre 40 y $50 \mathrm{cms}$., habiendo casos en que su grosor fue de sólo 20 a 25 cms. Su rendimiento fue del $25 \%$ en relación al primer grupo y en el valle llegaron a 20,000 has.

5. Suelos con menos de $25 \mathrm{cms}$. de grosor y con drenaje natural escaso. Fueron 1.500 has.en el valle y su rendimiento fue del $20 \%$ en relación al primer grupo. ${ }^{13}$

12. АНА, FCT, саja 7, exp. 51, foja 74 .

13. Comisión Nacional de Irrigación, Sistema Nacional de Riego "Presidente Calles", pp. 14-16. 


\section{EL VALLE Y SU IRRIGACIÓN}

Aguascalientes es un estado integrado por cuatro regiones naturales (las sierras y los valles) que delimitaron a los cuatro partidos político-administrativos. Por sus características geográficas y de infraestructura en comunicaciones el partido de la capital y el de Rincón de Romos fueron el granero natural del estado; sus agricultores contaron desde 1884 con el ferrocarril, lo que les permitió modificar sus estrategias productivas en vista del acceso a un mercado más amplio; además los ríos Santiago, Morcinique, Chicalote y Aguascalientes les permitieron regar en pequeña escala los extensos terrenos del valle central, por lo que el acceso a estos recursos fue fundamental para la modificación de las estrategias productivas en sus terrenos.

La temporada de lluvias abarca los meses de junio a octubre, pero es irregular. Los inviernos son secos y los veranos calurosos. Las lluvias en verano son favorables para el maíz y el frijol, pero el hecho de que no llueva en invierno impide los cultivos propios de la estación; vale decir que cuando se retrasa el temporal las siembras se inician tarde y las plantas son alcanzadas por las heladas tempranas de fines de octubre. ${ }^{14}$ Sólo una pequeña parte de las tierras de labor podían ser irrigadas con eficiencia por las presas, norias y bordos o estanques construidos en el cauce de los ríos.

En 1906 el nivel de concentración de la tierra en el estado fue extremo: de los 2,882 propietarios, 138 pagaron el $76.5 \%$ del impuesto catastral. El 68.6\% de la tierra estaba en manos de 36 familias $^{15}$.

14. Casi el $80 \%$ de las lluvias fueron en el verano, en primavera ocurrió el $5.3 \%$ y en invierno el $6 \%$. El promedio de precipitación anual en el valle no fue mayor a los $540 \mathrm{~mm}$. y la mayoría de los arroyos de los que se nutrieron los ríos no llevan agua en tiempos de secas. Véase: Comisión Nacional de Irrigación. Sistema Nacional de Riego "Presidente Calles". Op. cit., p. 8

15. Beatriz Rojas, op. cit., p. 96. 


\begin{tabular}{|c|c|c|c|c|c|}
\hline \multicolumn{6}{|c|}{$\begin{array}{l}\text { Fincas rústicas con capital mayor de } 5 \text { mil pesos, por } \\
\text { municipalidad y por calidad de tierra, según el catastro de } 1913\end{array}$} \\
\hline Municimo & NomERO & Rikco (has.) & Mrolo neco & Thuronal & A gostaderro \\
\hline Aguascalientes & 121 & 581 & 530 & 30,837 & 112,139 \\
\hline Jesús María & 25 & 138 & 305 & 3,602 & 46,570 \\
\hline Calvillo & 45 & 5 & 194 & 12,058 & 29,838 \\
\hline Rincón Romos & 13 & 20 & 1,021 & 10,800 & 89,037 \\
\hline S.José de Gracía & 8 & 0 & 178 & 943 & 19.326 \\
\hline Cosío & 4 & 48 & 91 & 2,017 & 6,527 \\
\hline Asientos & 13 & 133 & 376 & 5,818 & 36,577 \\
\hline Tepezalá & 7 & 0 & 60 & 3,164 & 14,847 \\
\hline TOTALES & 236 & 925 & 2,755 & 69,239 & 354,861 \\
\hline
\end{tabular}

Para 1913, entre los ocho municipios del estado, la cantidad total de tierras registradas fue de 427,780 hectáreas, de las cuales sólo 3,680 contaban con riego, es decir el 0.86\%; esto nos explica la necesidad de almacenar aguas para irrigación y nos da una mejor idea de la urgencia de construir presas para garantizar los cultivos. Es un hecho que las presas o bordos no fueron suficientes y que los beneficios del riego sólo alcanzaron a una reducida parte de los terrenos de cultivo en el valle.

La situación anterior fue observada por los gobiernos emanados de la lucha armada, en particular en lo referido al valle de Aguascalientes, ya que reunía características especiales: fertilidad mal explotada por la existencia del latifundio, trabajadores agrícolas capaces y no propietarios de tierras, poblaciones alrededor (las partes serranas y el valle del Tecuán) que dotadas en propiedad ejidal abastecían de mano de obra en los tiempos necesarios para los cultivos de las pequeñas propiedades del valle. Además, excelentes comunicaciones heredadas del Porfiriato y necesidad de financiamiento para obras de infraestructura agrícola que tal vez por su costo estuvieron fuera del alcance de los hacendados locales.

Observarían estos gobiernos un valle central apto para la explotación agrícola basada en la pequeña y la mediana propiedades, ro- 
deado de terrenos de menor calidad en donde se podrían promover las solicitudes de tierras en propiedad ejidal que completarían el marco de explotación agrícola propuesto en el artículo 27 de la Constitución de 1917: la convivencia entre la explotación moderna y la tradicional, la armonía entre rancheros y ejidatarios. Aquí sería posible experimentar la reorganización de la explotación agrícola dirigida desde el nuevo estado revolucionario.

Sin duda los objetivos de los revolucionarios fueron la destrucción del latifundio y la reducción del control de los hacendados sobre los recursos productivos. No obstante, no se trató de construir un país de ejidos sino de fraccionar las propiedades en pequeños lotes a la medida de las fuerzas de los agricultores individuales que con su esfuerzo abastecerían el mercado nacional.

Pero no sólo se atiende a las necesidades de los pueblos, sino que se acude también en apoyo y auxilio de los agricultores individuales que, siendo aptos para el cultivo agrícola, carezcan sin embargo, en virtud del monopolio latifundista a la sazón imperante. de una extensión pequeña o mediana de tierra a la que pudieran dedicar su trabajo y su esfuerzo creador. como colonos (si se trata de colonias cooperativas o de otra clase), o como agricultores o empresarios independientes (si se trata de la creación de nuevas empresas agrícolas. en forma de ranchos o granjas)... la pequeña propiedad -rancho o granja- para el ranchero de raza blanca o para el mestizo evolucionado; y la mediana propiedad. o sea la porción en lo que fuera latifundio, se deja en propiedad. al hacendado... hay que basar la economía agrícola... no en el ejido, incapaz de suyo para satisfacer ese postulado, sino en la pequeña y mediana propiedad, en la granja y el rancho que no llegue a constituir por su extensión un latifundio. Sólo el pequeño y mediano propietario disponen de capacidad y recursos suficientes para desarrollar un sistema de abundante producción. ${ }^{\text {lit }}$

La anterior anotación viene al caso porque en Aguascalientes hasta la década de los veinte las haciendas dominaron el territorio del valle, persistieron hasta 1925, año en el que el gobernador Eli-

16. Antonio Díaz Soto y Gama, La cuestión agraria en México, $2^{\mathrm{a}}$ ed., México, El Caballito, 1976, pp. 12-14. 
zalde empezó a afectar sus mejores tierras, ya para la creación de ejidos, ya para la colonización con pequeño productores.

En los años del movimiento armado no cambió la situación de los campesinos hidrocálidos. La mayoría de las familias rurales estuvieron sin tierra, en un estado temporalero que sólo en años afortunados logró que el río San Pedro tuviese la corriente suficiente para llenar las "norias" criadas a lo largo de su recorrido por el centro del valle y para irrigar con "ruedas morunas"1i algunas tierras de su ribera (por supuesto las cercanas a los cascos de las haciendas importantes del estado y a algunas pequeñas propiedades).

Con la construcción de grandes presas, canales y demás obras necesarias para la irrigación aumentarían los rendimientos y la seguridad de las cosechas. Según cálculos de la Comisión Nacional de Irrigación, para los mejores terrenos del valle de Aguascalientes los rendimientos en temporal de maíz eran de 300 a $500 \mathrm{~kg}$. en frijol de 100 a 200 kg. y en trigo de 600 a 800 kg. por hectárea; si se garantizara el riego, según la misma Comisión, se podrían obtener en maíz de 1,200 a 2,000 kg., en trigo de 1,000 a 1,500 por hectárea y 20 toneladas de alfalfa por la misma unidad de superficie. ${ }^{18}$

Antes de la construcción del sistema Presidente Calles hubo dos posibilidades para cultivar con riego: poseer terrenos cercanos a las márgenes de los ríos o poseer capital suficiente para construir pequeñas presas de almacenamiento. En el primer caso encontramos a hacendados y a pequeñas propiedades y en el segundo sólo a los hacendados.

17. En el sistema de riego por "ruedas morunas" el agua se tomaba por medio de túneles, caños, zanjas, tajos, o canales de derivación abiertos en los bordes de los ríos. así se llevaba el líquido a las fosas de las "norias", desde ahí se trasladaba el agua a los campos de cultivo por medio de ruedas con cubos movidas por tracción animal, por palancas, por balancines o por bimbaletes. Una rueda de 18 cubos proporcionó en el Municipio de Jesús María en 1907, 250 litros de agua por minuto. Este sistema también se utilizó para mover los molinos de harina, como en San Cayetano. 18. Comisión Nacional de Irrigación. Sistema Nacional de Riego "Presidente Calles"... op. cit., p. 16. 
La utilización de las aguas de los ríos por los propietarios de las tierras de sus vegas se basó legalmente en el decreto federal del 5 de julio de 1888, el cual reconocía los derechos de quienes habían usado con anterioridad las aguas. Ese fue el caso, por ejemplo, de la señora Domitila Aldana, dueña de los ranchos Las Norias y San Pascual. ${ }^{19}$

Por su parte, los hacendados utilizaron las aguas que pasaron por sus propiedades practicando la irrigación en escala relativamente amplia para la época en las haciendas de Natillas, La Punta, Santiago, Garabato, San Antonio, Pabellón, Letras y el Saucillo. Tuvieron las facilidades legales y económicas necesarias para construir pequeñas presas, como la que pretendía hacer la dueña de la hacienda de Santiago en 1919.

Conviene hacer notar que los propietarios, grandes o pequeños, que introdujeron obras de riego en sus fincas fueron pocos. La mayoría siguió dependiendo del errático temporal, como lo indica claramente el siguiente cuadro.

\begin{tabular}{|c|c|c|c|c|c|}
\hline \multicolumn{6}{|c|}{$\begin{array}{c}\text { Estadística de Superficies cultivadas, Aguascalientes, } \\
\text { noviembre de } 1923{ }^{20}\end{array}$} \\
\hline & \multicolumn{2}{|c|}{ SUPERFICIE WE RIECO } & \multicolumn{2}{|c|}{ SUPERRICE DE TEMPORAL. } & \multirow{2}{*}{$\begin{array}{l}\text { TOTAL. } \\
\text { (has) }\end{array}$} \\
\hline & (has) & 9 & (has) & $\%$ & \\
\hline Aguascalientes & 1.152 & 2.8 & 39,962 & 97.2 & 41,114 \\
\hline Rincón de Romos & 1.073 & 8.2 & 11.998 & 91.8 & 13,071 \\
\hline Asientos & 551 & 8.7 & 5,740 & 91.3 & 6,291 \\
\hline Calvillo & 510 & 3.2 & 15,573 & 96.8 & 16,083 \\
\hline Tepezalá & 125 & 4.4 & 2,071 & 95.6 & 2,826 \\
\hline San José de Gracia & 208 & 12.5 & 1.458 & 87.5 & 1,666 \\
\hline Jesús María & 279 & 5.4 & 4.860 & 94.6 & 5.139 \\
\hline Cosío & 139 & 7.2 & 1,802 & 92.8 & 1.941 \\
\hline TOTALES & 4.037 & 4.6 & 84.094 & 95.4 & 88,131 \\
\hline
\end{tabular}

19. AHEA. Fondo Juzgado de Distrito, (en adelante FJI) caja 24, exp. 44, mayo 21 de 1906.

20. AHEA, SGG, Caja 144, exp. 384.

100.1 C A A 
En 1923 de 88,131 has. de tierra de labor, 84,094 has. (el 95.4\%) se cultivaron en temporal y 4,037 has. (el 4.6\%) en riego, 357 has. más que en 1913. Los principales productos agrícolas fueron: maíz, frijol, chile, trigo y cebada. La costumbre fue sembrar maíz y frijol en la misma tierra apozolándolos (un surco cada uno); los rendimientos promedio en el municipio de Aguascalientes durante ese año fueron: en maíz de temporal 12 hectolitros por hectárea, en frijol 5 hectolitros por hectárea, en trigo $540 \mathrm{~kg} /$ hectárea y en chile 1,596 $\mathrm{kg} /$ hectárea.

A fines de los años veinte, en los estudios previos hechos por la Comisión Nacional de Irrigación para construir una presa sobre el río Santiago, se calculó que la demanda de agua para riego por hectárea fue de $5,100 \mathrm{~m}^{3}$ anuales; para satisfacer los requerimentos de las aproximadamente 40,000 hectáreas del valle se necesitarían almacenar en presas al menos 204,000,000 de $\mathrm{m}^{3}$ de agua. ${ }^{21}$

Para ver las posibilidades de cubrir la demanda anterior se hicieron cálculos de los escurrimientos de los ríos principales del valle y de las posibilidades de construir vasos que almacenaran sus aguas, se localizó en cada río el punto más adecuado para represar sus aguas y desde ahí se trazó la cuenca de captación; la del río Santiago se calculó en 653 km², la del río Pabellón en 334 km² (en este caso no se ubicó un punto adecuado para hacer una presa por lo que se optó por sugerir que por medio de un túnel de desviaran sus aguas al vaso del río Santiago), la del río Morcinique en 190 km² y la del río Aguascalientes en $425 \mathrm{~km}^{2}$.

La Comisión Nacional de Irrigación concluyó que los ríos podrían proporcionar agua para irrigar hasta 34,810 hectáreas de la siguiente manera: el río Santiago 13,750, el río Pabellón 5,840, el río Morcinique 5,220 y el río Aguascalientes 10,000. Si bien la superficie dicha no alcanzaría la totalidad calculada para el valle, sería sufi-

21. Comisión Nacional de Irrigación. Sistema Nacional de Riego "Presidente Calles", op. cit., p. 11.

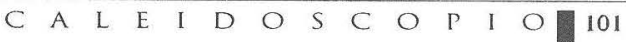


cientes para revolucionar la eficiencia productiva de la región central del estado. ${ }^{22}$

\section{LOS BENEFICIARIOS POTENCIALES}

En 1923 había 283 agricultores en el municipio de Aguascalientes, 53 en Asientos, 1,238 en Calvillo, 74 en Cosío, 300 en Jesús María, 99 en Rincón de Romos, 19 en San José de Gracia y 24 en Tepezalá. En este mismo año, la sequía, las heladas tempranas, el granizo y el chahuixtle provocaron grandes pérdidas en los cultivos. Los 2,090 agricultores cultivaron 88,131 has, de las cuales 4,037 (4.6\%) fueron con riego y 84,094. (95.4\%) de temporal..$^{23}$

En los municipios de Asientos y de Tepezalá, en los que el clima es semifrío y la altitud rebasa los 2,000 metros sobre el nivel del mar, la presa beneficiaría sobre todo las tierras de la hacienda de San Antonio. En la parte sur del municipio de Cosío se localizaron los terrenos de la hacienda de La Punta, que fue el límite norte de las tierras que irrigaría la presa. Al municipio de Calvillo la construcción de la presa no le beneficiaría directamente, incluso la podría perjudicar en el hipotético caso de que perdiesen mercado sus productos agrícolas.

Al municipio de la capital la presa le beneficiaría una buena parte de sus tierras, sobre todo en el valle cuyas suaves pendientes habían hecho posible cierto desarrollo de la agricultura mecanizada. En el valle estaban de hecho situadas algunas de las haciendas más importantes de la región: Agostaderito, Cañada Honda, Cieneguilla, La Escondida, La Guayana, Jaltomate, Loreto, Ojocaliente, Palo Alto, Peñuelas, San Bartolo, Santa María, El Zoyatal, Chicalote, La Cantera y Hacienda Nueva.

22. Idem, pp. 41-56.

23. AHEA, RF, caja 144, exp. 385, año de 1923. El incremento en el número de has. de riego con respecto a 1913 es menor.

102 C A 
En el municipio de Rincón de Romos, en donde estaban ubicadas las haciendas de Pabellón y El Saucillo, dos de las más importantes de todo el estado, la presa dejaría sentir también sus benéficos efectos.

En la cabecera del municipio de San José de Gracia, un antiguo pueblo de indios, encontramos la gran paradoja de la construcción de la presa. Situado en la parte alta de la sierra de Pabellón, fue inundado por las aguas y tuvo que ser reubicado. Hay que señalar que a la fecha no existe un estudio antropológico que detalle los efectos que tuvo dicho traslado.

\section{LA CONSTRUCCIÓN DE LA PRESA}

Plutarco Elías Calles ha sido recordado en Aguascalientes por el conflicto cristero y por la creación del Partido Nacional Revolucionario; sin embargo, hay que reconocerlo como el modernizador de los sistemas de producción agrícola del valle de Aguascalientes: promovió la afectación de tierras y ensayó su política agrícola moderna a través de la Comisión Nacional de Irrigación. Su reforma agraria no significó sólo reparto de tierras, sino el uso de máquinas modernas, la construcción de presas y la capacitación de los nuevos agricultores.

El gobierno federal, inspirado por el modelo americano de las granjas agrícolas o farmers, se propuso modernizar la producción e impulsar el desarrollo de la pequeña propiedad. En Aguascalientes, estado dominado aún por la hacienda, la modernización implicaba la posibilidad de atender las demandas de las familias que carecían de tierras y no tenían acceso al crédito ni a los sistemas de riego.

El llamado "ranchero emprendedor" fue el beneficiario de la modernización callista y el encargado de demostrar que la gran propiedad no era ya la más eficiente, ni la más adecuada para las necesidades del país. Los propietarios en pequeño fueron el modelo 
a seguir y el colchón que amortiguó los ambiciosos afanes de los terratenientes y de los solicitantes de tierras en propiedad ejidal. ${ }^{24}$

En Aguascalientes la "irrigación revolucionaria"25 y la promoción desde el Estado de la reforma agraria me permiten evaluar, al menos en las mejores tierras del estado (el valle central), el impacto de la modernización callista. El desarrollo de la reforma agraria y del primer gran proyecto de irrigación afectaron las formas de convivencia agraria, pues reordenaron la propiedad, redujeron el control de los terratenientes y colaboraron en la reorganización del poder a nivel local. Al afectar la propiedad de la tierra y el acceso al agua, la estrategia callista redujo el poder terrateniente y sentó las bases para nuevas relaciones agrarias y productivas en la zona.

Lo anterior fue en buena medida resultado de la creación de la Comisión Nacional de Irrigación: a partir de 1921 y debido al aumento de solicitudes para usar agua para riego en el país y a los intereses que se afectaban al conceder los permisos para la utilización de las aguas de propiedad federal, se creó, dentro de la Secretaría de Agricultura y Fomento, la Dirección de Irrigación, la cual funcionó entre 1921 y $1923 .^{26}$ En 1924 esta dependencia se transformó en Departamento de Reglamentación e Irrigación y en 1926, bajo mejores augurios, en Comisión Nacional de Irrigación. Al respecto de esta última se dijo:

Por fin ha llegado la época de las construcciones que no tratan sólo de resolver el problema de una hacienda o de un conjunto de ranchos, sino que

24. Enrique Krauze, Historia de la Revolución Mexicana 1924-1928. La reconstrucción económica. México, El Colegio de México, 1977.

25. Por "irrigación revolucionaria" entiendo la política promovida desde 1926 por la Ley Federal de Irrigación. Una explicación amplia de su utilización para el caso del Río Conchos en Chihuahua se puede ver en Luis Aboites Aguilar, La Irrigación Revolucionaria. Historia del Sistema Nacional de Riego del Rio Conchos, Chihuahua, 1927-1938. México, SEP/CIESAS, 1988.

26. Comisión Nacional de Irrigación, La obra de la Comisión Nacional de Irrigación durante el Régimen del Sr. Gral. de División Lázaro Cárdenas 1934-1940, México, s.n.e., 1940. pp. 15-17.

104 C C A A L L E E 
toman en cuenta regiones enteras, que implican la construcción de obras cuya magnitud no había sido ni siquiera intentada en décadas pasadas... es la era de la Comisión Nacional de Irrigación. ${ }^{2 i}$

La Comisión logró que se abrieran nuevas tierras al cultivo y, a través de la construcción de grandes sistemas de riego, procuró para el "ranchero revolucionario" (la copia mexicana del granjero americano) las condiciones óptimas para producir, ampliar la frontera agrícola, mecanizar las labores e incrementar la productividad. Además, al afectar la estructura de la propiedad de la tierra y el agua, promovió la presencia de nuevos actores.

La Ley de Irrigación con Aguas Federales de 1926 eliminó los privilegios que tenían los grandes propietarios en materia de acceso al agua. En su artículo 2º la ley declaró de utilidad pública la irrigación de las propiedades agrícolas privadas, "cualesquiera que sean su extensión y cultivos", siempre que fuesen susceptibles de aprovechar aguas de jurisdicción federal. Para las grandes haciendas del valle de Aguascalientes lo anterior significó la posibilidad de que se les expropiasen el agua y sus mejores tierras, y por lo tanto la devaluación de su propiedad, la reducción de su importancia económica, de su capacidad para controlar la producción y de comercializar los productos agrícolas del valle. A partir de la promulgación de la ley, la capacidad productiva de las haciendas del valle estaría condicionada por los planes político-agrícolas nacionales representados en 1926 por Plutarco Elías Calles.

Con su artículo $3^{\circ}$, la ley creó un órgano encargado de promover y construir las obras de riego en la república: la Comisión Nacional de Irrigación. Con ella la realización de los proyectos y los recursos financieros serían administrados directamente por el gobierno federal en detrimento de las élites locales. De hecho, en el caso de la construcción de la presa en el río Santiago se separó a los hacen-

27. Francisco Gómez Pérez, "La irrigación en México", en Irrigación en México, Mayo y Junio de 1940, p. 10. 
dados locales de la dirección de las obras y con ello de su posible utilización, comercialización y control de los terrenos irrigados. Se modificó sustancialmente el proyecto inicialmente planteado por el Ing. Medina Ugarte en 1897.

La Comisión estudiará las iniciativas de riego que juzgue convenientes desde el punto de vista económico y social. formulará los proyectos correspondientes y los dará a conocer a los propietarios de las tierras beneficiadas. Si éstos lo encuentran conveniente para sus intereses particulares, deben emprender las obras en un plazo determinado: pero si se oponen a ello la Comisión resolverá si deben o no ejecutarse las obras. En caso afirmativo éstas serán pagadas con tierras de regadío que pasen al dominio del gobierno federal. ${ }^{28}$

El artículo $5^{\circ}$ estableció que los propietarios de tierras dentro del perímetro de irrigación de los Sistemas de Riego, estarín obligados a compensar al gobierno federal los gastos erogados en la construcción de las obras de infraestructura de riego. El pago sería calculado de tal suerte que el propietario conservase una cantidad de tierras dentro de los sistemas de riego cuyo valor fuese igual al que tenían antes de la ejecución de las obras. En la práctica, ello significó la capacidad legal del gobierno federal para reducir la superficie de las grandes propiedades. Las haciendas de Pabellón, El Saucillo, Garabato, San Luis de Letras y Santiago fueron las que, en Aguascalientes, resintieron los efectos de la ley.

En el artículo $13^{\circ}$ inciso "b" de la citada ley, se estableció que las concesiones otorgadas con anterioridad a 1926 para utilizar en riego las aguas de los ríos y que impidiesen la distribución de las aguas en los términos de la nueva ley serían modificadas de acuerdo con ésta. Es decir que todas las concesiones de las haciendas del valle se derogaron y se supeditaron a un nuevo convenio con el gobierno federal. Este hecho no benefició a los antiguos concesionarios dado el momento crítico de la política estatal en donde los hacendados lo-

28. "Antecedentes y desarrollo de la irrigación en México", en Irrigación en México, número 3. volumen XXI, noviembre y diciembre de 1940, p. 13. 
cales estuvieron perdiendo sus espacios de poder político heredados del porfiriato.

Además, vale la pena agregar que las haciendas estuvieron amenazadas por las solicitudes de dotación ejidal y que las respuestas favorables dependieron de la orientación política del momento. Nuevamente es ilustrativo el caso de las haciendas del Saucillo, Pabellón y Santiago, cuyos dueños perdieron su categoría de "hacendados" y pasaron a ser considerados por la Comisión Nacional de Irrigación como "simples usuarios" de las aguas del sistema de riego "Presidente Calles".

En el caso de la hacienda del Saucillo, ${ }^{29}$ la afectación de la finca tuvo particular importancia por el hecho de que de las 2,707 hectáreas afectadas en favor de Rincón de Romos, el $75 \%$ tuvo por "derecho de accesión" el privilegio de usar las aguas almacenadas en la presa de la hacienda. Según la ley, la posesión definitiva comprendió "todas las accesiones, costumbres y servidumbres de las tierras dotadas"; además precisaba que "si dentro de los terrenos con que fue dotado el pueblo existe una extensión de regadío, el agua, por derecho de accesión, deberá tomarse necesariamente de donde siempre se ha tomado". Lo anterior autorizó al ejido de Rincón de Romos a tomar agua de la presa de El Saucillo en beneficio de sus tierras.

La hacienda del Saucillo tuvo sus mejores tierras situadas al lado sur, y justo ahí fueron afectados por la resolución presidencial. Al ocuparse estos terrenos se destruyó necesariamente la unidad agrícola que representó la finca, ya que en sus terrenos del lado norte no fue posible la agricultura ni se contó con sistemas de riego. Con dicha resolución se afectaron también los canales de irrigación y demás obras de captación y conducción de agua; en resumen, se nulificó por completo la potencia económica de la finca, así como su lote de maquinaria.

29. La información utilizada en esta parte corresponde a: AHEA, FJD, Cajas 42,53 , 60 y 67, año de 1926. 
La hacienda de Santiago, según el contrato suscrito con la Comisión Nacional de Irrigación el 19 de noviembre de 1929, tuvo 998.5 hectáreas en la primera unidad del sistema de riego 01, propiedad de la sucesión de la Sra. Ascensión López viuda de de la Vega. Además, cedió 661.8 hectáreas ubicadas en la primera unidad al gobierno federal, a cambio de las cuales la Comisión Nacional de Irrigación le otorgó concesión para regar 171 hectáreas de los terrenos que conservaron en la primera unidad.

Sin embargo, el 28 de diciembre de 1930 se avisó a Concepción de la Vega que se suspendería el suministro de agua a partir del $l^{\circ} \mathrm{de}$ enero de 1931. La razón aducida por la Comisión de Irrigación fue que la hacienda no tenía aún acreditado el derecho a usar dichas aguas debido a que el contrato-concesión no se elevó al rango de escritura pública dentro de los plazos fijados por la ley. Un par de años después, en septiembre de 1933, los vecinos de la hacienda de Santiago solicitaron al gobierno estatal dotación de tierras en propiedad ejidal señalando como única finca afectable a la propia hacienda. El $1^{\circ}$ de noviembre de 1934 se levantó el censo, encontrándose a 216 habitantes, de los cuales 62 tuvieron derecho a tierras. La finca conservó sólo 1,047.6 hectáreas, repartidas en 5 fracciones de desigual tamaño.

Sin embargo, el gobierno ignoró los términos en que se hizo el fraccionamiento, argumentando que las escrituras ccrrespondientes no se habían inscrito en el registro público de la propiedad antes de la resolución presidencial y que no se había demostrado que el legatario estaba muerto antes de la publicación de la solicitud de tierras. Por lo tanto y para los efectos de la solicitud ejidal, se tomó a la hacienda de Santiago como propiedad indivisa con la calidad y extensiones que encontró el ingeniero comisionado por la Comisión Local Agraria.

Se formó así el ejido de Santiago con 62 parcelas de 4 hectáreas de riego cada una (248 hectáreas), 1 parcela escolar de riego y terrenos pastales y de monte para usos comunales a razón de 10 hectá- 
reas cada uno de los 62 dotables (620 hectáreas). En total fueron 872 has., lo que dejó a la hacienda con sólo 502 has. Además para irrigar las 252 has. proyectadas en la dotación se fijó un volumen anual por ha. de $10,000 \mathrm{~m}^{3}$, que en total dio 2,520,000 $\mathrm{m}^{3}$ de agua anuales. El agua se tomaría del río Santiago por la toma establecida por la hacienda y de los canales de la Comisión Nacional de Irrigación.

La hacienda de Santiago desapareció como tal en 1935, cuando se dio posesión provisional al poblado del mismo nombre, el cual usufructúa desde entonces las tierras de la hacienda, quedando las antiguas propietarias sin recurso agrícola e imposibilitadas para pagar la cantidad que le adeudaban al distrito de riego. Por todo lo anterior el gerente del distrito solicitó que se les condonara la deuda. Por su parte las propietarias se quejaron del trato recibido en la carta enviada por María Guadalupe de la Vega al gerente de colonización del Distrito de Riego de Pabellón:

...nuestras propiedades rústicas han sido repartidas con exceso y a diversos pretextos, en algunas ocasiones a base de repartos ejidales, en otras por encontrarse ociosas, lo mismo nos han desposeído de tierras de riego, temporal o cerril, nos parece que lo que se busca y se ha encontrado es dejarnos en la miseria, nuestra insolvencia es tan manifiesta que en diversas ocasiones hemos tenido que despojarnos de ciertas prendas de uso diario para enajenarlas y con su producto tener que comer... Después de varias afectaciones hechas a la hacienda nos quedó la pequeña propiedad consistente en la huerta, en los últimos años ha dado muy bajo rendimiento debido a que el Distrito no nos ha proporcionado agua para riego de dicha huerta ni para los usos públicos y domésticos del casco de la hacienda. ${ }^{30}$

Para el caso de la hacienda de Pabellón tenemos que Manuel Azanza, su dueño, solicitó el 21 de julio de 1926 la protección federal contra la afectación del agua de su hacienda para dotar a los ejidatarios de San Francisco de los Romo. Alegó en su favor que había estado al corriente de sus pagos por el uso de las aguas del río Pa-

30. Archivo Histórico del Distrito de Riego 01. Pabellón de Arteaga, Ags., sin clasificar y en peligro de desaparición. 
bellón contenidas en su presa desde tiempo inmemorial, de afectársele el agua se impediría el cultivo de aproximadamente 900 hectáreas con cultivos de chile, maíz, trigo y frutales, importantes para abastecer el mercado local.

Los alegatos no fructificaron y el 3 de enero de 1931 la hacienda de Pabellón acordó ceder a la Comisión Nacional de Irrigación 1,532.6 has. en compensación por los gastos erogados en la construcción del Sistema de Riego 01. Conservó dentro de la primera unidad y con derecho a riego sólo 682 has. y hasta $3,220,000 \mathrm{~m}^{3}$ de agua anuales. En este convenio no se incluyó la presa y demás obras de riego.

El 4. de marzo del mismo año se modificó el convenio anterior y la hacienda tuvo que ceder al gobierno federal 689 hectáreas dentro de la primera unidad y 4,000 más de agostadero, lo que incluía la huerta, la presa y demás obras de riego. El precio que se fijó a las tierras fue de 175,000 pesos. En total la comisión adquirió 2,221.7 hectáreas dentro de la primera unidad del sistema de riego y 4,000 fuera de ella.

\section{COMENTARIO FINAL}

En la década de los 20's, el desarrollo de la agricultura en el valle de Aguascalientes tuvo que ver con la reducción del poder de las haciendas y dicho poder, sin lugar a dudas, se basó en el control de la tierra y del agua. El dominio que tuvieron las haciendas en el centro del valle se redujo con acciones como el reparto agrario y la construcción de la presa Calles.

La modernidad tomó forma en los procesos de afectación a las haciendas. El vacío de poder que dejó la hacienda fue ocupado inmediatamente por el gobierno federal, que sentó las bases para un nuevo sistema de dominación agraria y de producción agrícola. Quedó claro que luego de los procesos anteriores en el valle existieron ex-hacendados, peones, rancheros y ejidatarios; los dos últi- 
mos con todo el apoyo de los gobierno estatal y federal. No cabe duda que los tiempos de la hacienda habían terminando.

Es así que, cuando se inicia el aprovechamiento del sistema de riego Presidente Calles, las tierras beneficiadas ya no estaban concentradas por un solo dueño: propietarios medios, peones y ejidatarios estuvieron en condiciones de disputar el uso y aprovechamiento del agua a los hacendados. Fue el momento de intentar completar la reforma agraria, de llevarla a su fase productiva, de promover el modelo callista del campesino medio modernizado con el apoyo del gobierno federal.

La propuesta de modernización de la agricultura buscó derribar el control latifundista apoyando a pequeños propietarios y ejidatarios; el control del agua de riego tuvo aquí un papel fundamental. Tal y como se dijo oficialmente:

era necesario dar un vigoroso impulso a la agricultura, ampliando esta importante fuente de riqueza; pero observó que era absolutamente necesario el apoyo oficial para llevar a cabo la construcción de obras de riego, con lo que, por otra parte, se daría ocupación a un considerable número de trabajadores. De ahí surgió la ley de irrigación. ${ }^{31}$

El propósito abierto de la Comisión Nacional de Irrigación era el de crear una clase media campesina, más alta que la del ejidatario por tener mayores iniciativas, ambiciones. experiencias y recursos, clase que serviría para emular, para ejemplo y como enseñanza, a la de los pequeños campesinos. Como ejemplo de esa clase media campesina se mencionaba a la de los agricultores. en 1926, de La Laguna y el Mante en Tamaulipas.

Lo anterior fue la concreción de la estrategia callista para revolucionar la agricultura. A decir de Beatriz Rojas, la ley

suponía mayor productividad, colonización, privilegiar a los agricultores independientes, debilitar las haciendas, favorecer al ranchero y campesino medio y desarrollar la agricultura capitalista al estilo del norte californiano. ${ }^{33}$

31. Comisión Nacional de Irrigación, op. cit., p. 17.

32. Adolfo Orive Alba, La Irrigación en México, México, Grijalbo, 1970, p. 70.

33. Beatriz Rojas, op. cit., p. 125. 
Los rancheros del estado de Aguascalientes habían venido utilizando en general métodos rudimentarios que, junto al mal temporal, terminaron normalmente en malas cosechas. Además, en ocasiones ni siquiera los arcaicos instrumentos (arados, semillas y yuntas) eran los adecuados para el tipo de cultivo y la cantidad de terreno. Plutarco Elías Calles propuso a las clases medias agrarias como puntales de la moderna agricultura: los medieros, los arrendatarios y los pequeños propietarios deberían dejar de ser explotados por los dueños de las haciendas y pasar a ocupar asiento de primera fila en la reconstrucción nacional; de ellos provendría el empuje necesario para el desarrollo de la agricultura.

Su propuesta no era pulverizar la propiedad de la tierra, sino que pensó en la cantidad que un trabajador puede hacer producir con créditos y técnicas modernas. El fraccionamiento de los latifundios se guió por esta idea: no sólo los improductivos, sino todos los que no se adecuaran a las exigencias del mercado nacional se verían afectados.

El problema agrario, entendido y resuelto como lo concibo yo, como un problema integral y constructivo que comprende la dotación de tierras, la creación y formato de la pequeña propiedad, el aprovisionamiento de aguas y la fundación del crédito agrícola que permita el fomento de la agricultura verdaderamente nacional, lejos de ser un programa suicida, es obra eminentemente constructora de bienestar y riqueza... ${ }^{34}$

No obstante lo anterior, y como ya lo hemos tratado de mostrar con el presente texto, el fraccionamiento de los latifundios tuvo más intereses que los mencionados en el discurso prsesidencial: afectar la tierra y el agua del Saucillo, Pabellón, San Antonio, Santiago y Letras significó eliminar las bases del control ejercido por

34. Carlos Macías (prólogo, selección y notas), Plutarco Elías Calles. Pensamiento político y social. Antología (1913-1936), México, INEHRM/FCE/Fideicomiso Archivos Plutarco Elías Calles y Fernando Torreblanca, 1988, p. 96.

112 C 
los hacendados y proponer al Estado, a sus instituciones y beneficiarios para ocupar los espacios del poder rural local. Por ello y a pesar de estar consideradas como ejemplo productivo estatal, las haciendas del valle se vieron atropelladas por la avalancha del poder central y su estrategia agrícola centralista y modérnizadora.

Los propósitos callistas fueron aceptados por la clase media rural, la cual estuvo preparada para el cambio. Estos trabajadores agrícolas de toda la vida necesitaron el apoyo del gobierno federal para sacudirse el poder de los hacendados y tener mejores expectativas de producción. El discurso presidencial y las condiciones reales se conjugaron en el valle originando el proyecto de modernidad agrícola de los treintas en Aguascalientes. Los resultados productivos y políticos de lo hasta aquí expuesto, se evaluarían ya en el periodo cardenista, en el cual muchas de las proyecciones aquí comentadas se verían sustancialmente modificadas. Pero la nueva situación tendrá que analizarse en un trabajo posterior. 
